\title{
Developmental Coordination Disorder
}

\author{
Daniel Brady ${ }^{1,2}$ \& Hayley C. Leonard ${ }^{2}$
}

1. School of Psychology and Clinical Language Sciences, University of Reading

2. School of Psychology, University of Surrey

\section{Abstract}

Developmental coordination disorder is a neurodevelopmental disorder primarily characterised by motor coordination significantly below that expected for an individual's age, in the absence of neurological or intellectual deficits (American Psychiatric Association, 2013). This poorer coordination has a significant negative impact on activities of daily living and individual wellbeing. While it is understood that the root cause of DCD likely lies in the development of the brain, there is presently no consensus into the precise nature of this neurological basis of the disorder. The aim of this chapter is to outline the current understanding of DCD from a developmental cognitive neuroscience perspective. It begins by briefly describing the presentation of DCD, before moving on to outline neuroscientific hypotheses and the evidence supporting them. The chapter concludes with an exploration of current issues in the field and potential future directions for research into the developmental cognitive neuroscience of DCD.

Keywords: Developmental coordination disorder, EEG, fMRI, TMS, DTI, motor development, neurodevelopmental disorders

\section{What is Developmental Coordination Disorder?}

Developmental Coordination Disorder (DCD) is characterised by motor competence significantly below that expected for an individual's age. The actual presentation of the disorder is somewhat heterogeneous, with different individuals presenting different profiles of impairment. However, 
common core features include: slower and less accurate motor performance, impaired balance, difficulty in motor learning, and poorer sensorimotor coordination (Geuze, 2003; Wilson et al., 2012). These core features result in more functional difficulties in activities of everyday living, such as: writing and drawing, dressing, using utensils, catching, and running (Summers, Larkin and Dewey, 2008). The full diagnostic criteria are presented in Table 1. All criteria should be met for a diagnosis of DCD.

Table 1. Diagnostic criteria for DCD - DSM-5 (American Psychiatric Association, 2013)

A Acquisition and execution of coordinated motor skills are below what would be expected at a given chronological age and opportunity for skill learning and use; difficulties are manifested as clumsiness (e.g., dropping or bumping into objects) and as slowness and inaccuracy of performance of motor skills (e.g., catching an object, using scissors, handwriting, riding a bike, or participating in sports)

B The motor skills deficit significantly or persistently interferes with activities of daily living appropriate to the chronological age (e.g., self-care and self-maintenance) and impacts academic/school productivity, prevocational and vocational activities, leisure, and play.

C The onset of symptoms is in the early developmental period ${ }^{a}$

D The motor skills deficits cannot be better explained by intellectual disability or visual impairment and are not attributable to a neurological condition affecting movement (e.g., cerebral palsy, muscular dystrophy, or a degenerative disorder)

${ }^{a}$ signs usually evident before school age

As well as these core symptoms, DCD has also been associated with negative secondary outcomes in a number of domains, including: cognitive (e.g. Leonard and Hill, 2015; Leonard et al., 2015), mental and physical health (e.g. Cairney et al., 2005; Faught et al., 2005; Piek et al., 2007; Pratt and Hill, 2011), social/interpersonal (e.g. Skinner and Piek, 2001; Miyahara and Piek, 2006), 
and academic domains (e.g. Miller et al., 2001; Watson and Knott, 2006; Wocadlo and Rieger, 2008).

It is currently estimated that DCD has a prevalence in school-aged children of approximately 5-6\% (American Psychiatric Association, 2013). This prevalence is also thought to be consistent across races and socio-economic statuses (Blank et al., 2012). Studies indicate that boys are twice as likely to show motor difficulties that can be classified as DCD (Lingam et al., 2009). It is recognised that DCD is not just a childhood disorder, and that a large proportion of children with the disorder will continue to experience problems in adolescence and adulthood (Cousins and Smyth, 2003; Kirby, Sugden and Purcell, 2014).

\section{The developmental cognitive neuroscience of DCD}

While diagnostic criterion D states that DCD is not attributable to a known neurological condition that affects movement, it is generally acknowledged that the root cause of the disorder lies in the brain. Early hypotheses posited that the cause is some form of brain damage (Gubbay et al., 1965); however, the current understanding is centred on the idea that brain development follows an atypical trajectory (Wilson et al., 2012). This section will provide an overview of neuroimaging research that has been conducted in DCD, beginning with investigations of the neural correlates of motor performance in individuals with and without the disorder. Next, we will cover studies examining neural correlates of DCD that were inferred from performance deficits observed in behavioural experiments ('cognitive hypotheses’; Wilson et al., 2012). These hypotheses fit broadly into the following categories: Visual perception, visuospatial attention, executive functioning, internal modelling deficits, and imitation (See Wilson et al., 2017, for a recent systematic review). Finally, more recent investigations of structural differences in DCD will be considered. 


\section{Neural correlates of motor performance}

Given that motor difficulties are core to the disorder, the majority of research investigating the neural correlates of DCD has explored some aspect of motor performance. These include: gross motor control, visually-guided fine motor control, motor learning, and timing.

\section{Gross motor control}

A number of different paradigms exist to directly explore aspects of gross motor control, including two-step reaching and visuomotor adaptation tasks (e.g. Kagerer et al., 2004, 2006; Hyde and Wilson, 2013). Unsurprisingly, children with DCD show atypical performance on these tasks, with slower initiation of movement and more errors in the two-step reaching task (Hyde and Wilson, 2013) and less sensitivity to smaller perturbations in the adaptation tasks (Kagerer et al., 2004, 2006).

Pangelinan, Hatfield, and Clark (2013) employed a simple reaching paradigm to explore motor control and its neural correlates in children with DCD. The task required participants to make rapid movements from the centre of a graphics tablet to one of two points located diagonally. In order to investigate the neural correlates, electroencephalography (EEG) was recorded throughout the experiment, and analysed using both event-related potential (ERPs; specifically the movementrelated cortical potential, MRCP) and spectral decomposition (focussed on alpha (7-13 Hz) and beta (13-30 Hz) bands) approaches. Although no behavioural differences were found between groups, EEG analyses revealed that the DCD group showed a greater mean amplitude in the $\mathrm{Fz}$ and $\mathrm{Cz}$ electrodes before movement onset, after accounting for age. The spectral analysis showed no changes in alpha or beta activity associated with age, and no differences between groups. Given that there is evidence that localises the source of the MCRP component to motor areas (specifically the sensorimotor and supplementary motor areas), the authors suggest that the DCD group require more cortical resources than their typically developing peers to execute these movements. 
Motor overflow is another presentation of poor gross motor control, and is typically defined as "the presence of extraneous movements occurring in parts of the body not actively involved in the performance of a task" (Licari et al., 2015, pp. 3-4). It is fairly common in young children but has also been observed in older children with DCD (Licari, Larkin and Miyahara, 2006; Licari and Larkin, 2008). Licari and colleagues (2015) explored the neural correlates of motor overflow in DCD using functional magnetic resonance imaging (fMRI), recording the Blood Oxygenation Level Dependent (BOLD) signal while the children undertook a task. The BOLD signal represents changes in the blood oxygen level across the brain and is an indirect way of measuring which particular areas are active during tasks (for a more complete explanation see Soares et al., 2016). Children with and without DCD performed two tasks with their dominant hand: a repetitive finger sequencing task and a repetitive hand clenching task. Overflow activity in their non-dominant hand was monitored using a motion-sensitive glove. Children with DCD demonstrated greater overflow than the control group for both tasks, particularly in the finger sequencing task. This task was also associated with greater activation in the left superior and left inferior frontal gyri than the DCD group. Furthermore, the DCD group showed increased activation in the right post-central gyrus. This suggests that over-activation in frontal areas and potential dysfunction in inter-hemispheric inhibition may play a role in motor overflow in DCD.

\section{Visually-guided fine motor control}

In addition to gross motor control, deficits in fine motor control have been identified in DCD. This typically manifests in activities of daily living such as poorer handwriting, which has been explored experimentally (e.g. Smits-Engelsman, Niemeijer and Van Galen, 2001). However, beyond these studies there appear to be very few purely behavioural investigations examining visually-guided fine motor control.

Thus far, three studies have looked at the neural correlates of visually-guided fine motor performance in children with DCD, employing tasks where participants used a joystick to either 
move a cursor to keep pace with a moving target (Kashiwagi et al., 2009) or to follow a trail while remaining within the lines (Zwicker et al., 2010, 2011). Kashiwagi and colleagues (2009) reported lower BOLD activity in the left superior and inferior parietal lobes and the left post central gyrus for children with DCD compared to controls, and the poorer performance on the task in DCD compared to controls also correlated with BOLD activity in the inferior parietal cortex. This led the authors to suggest that poorer performance on the task could be related to dysfunction in the posterior parietal cortex (PPC). While there may be an overall difference in BOLD activity between the two groups in this study, the reported poorer performance on the behavioural measure and the correlation with the BOLD response appears to be driven by a single participant in the DCD group with a particularly poor score. Consequently, the authors' conclusions should be treated with caution.

A wider range of atypical functional activity were reported by Zwicker and colleagues (2010, 2011), despite demonstrating no behavioural differences in the trail-following task between children with DCD and controls. Specifically, the DCD group showed greater BOLD activity than the control group in nine regions mostly located in the right hemisphere, including: the middle frontal gyrus, supramarginal gyrus, lingual gyrus, parahippocampal gyrus, posterior cingulate gyrus, precentral gyrus, superior temporal gyrus, and cerebellar lobule VI, as well as the left inferior parietal lobule. In contrast, the control group showed greater activation than the DCD group in five sites primarily located in the left hemisphere, including: the precuneus, superior frontal gyrus, inferior frontal gyrus, and post-central gyrus, as well as the right superior temporal gyrus (Zwicker et al., 2010). After practising the task for three days outside the scanner (Zwicker et al., 2011), behavioural performance did not change from baseline or differ between DCD and control groups, but the authors reported statistically significant Group by Time interactions for a number of cortical areas, including: the inferior parietal lobules bilaterally, the left fusiform gyrus, the right lingual gyrus and the right middle frontal gyrus. In addition, three cerebellar areas also showed an interaction: The right crus I, left lobule VI, and left lobule IX. However, no main effects for time or 
group are reported for these analyses, nor any post-hoc tests, making it difficult to interpret these results for the DCD group.

\section{Fine motor sequence learning}

As well as motor control, research into DCD has investigated motor learning. The results of this research have been mixed, with some studies suggesting that there is a motor sequence learning deficit in DCD (Gheysen, Van Waelvelde and Fias, 2011) and others suggesting that this deficit is task-specific (Lejeune et al., 2013).

In order to investigate the neural correlates of motor learning in children with DCD, Biotteau and colleagues (2017) employed a finger tapping sequence task. Two sequences were used: an over-learned sequence, which had been practised for fifteen days before the scanning session, and a novel sequence. Motor learning was assessed through accuracy on producing the over-learned sequence when concurrently completing a picture-naming task. Performance during the dual task was poorer than on the over-learned task alone, suggesting that it had not become automatic. Imaging revealed that there was higher activity in the right caudate and right insula during performance of the novel sequence. The authors note that this difference is not surprising given evidence suggesting recruitment of this area during the early stages of motor learning. However, the lack of a typically developing reference group makes it impossible to determine whether the profiles of activity presented are actually atypical.

\section{Timing}

Timing is an essential component of efficacious movement control. Individuals with DCD have been reported to have poorer timing ability compared to age-matched controls (Rosenblum and Regev, 2013) but, like most aspects of motor performance in DCD, the neural cause is not clear. Nonetheless, two studies have suggested that frontal areas involved in accurate timing may have reduced activity and connectivity in children with DCD compared to controls. 
In the first of these studies, children responded to a visual stimulus that was presented at high or low frequency by either tapping in synchrony or in syncopation with it (de Castelnau et al., 2008). Coherence in alpha and beta frequency band EEG activity between specific frontal, central, and parietal electrodes was recorded during the task. The DCD group showed more performance variability than the TD group, particularly in the high presentation frequency condition. The younger children with DCD displayed less frontal-central coherence than their TD counterparts; however, this difference diminished with age: the older children demonstrated similar levels of alpha and beta activity compared to age-matched controls.

In the second study, children responded to a stimulus presented on screen with either predictable or unpredictable inter-stimulus intervals (Debrabant et al., 2013). Reaction times decreased and the proportion of anticipatory responses increased in the predictable compared to the unpredictable timing conditions for controls, and greater activation was recorded in the right middle and inferior frontal gyri for the unpredictable timing condition over the predictable timing condition. In contrast, the DCD group demonstrated no differences in either behavioural performance or BOLD activity between the two conditions. When the two groups were compared on activity differences between the two conditions, greater activation of the middle frontal gyrus, temporal-parietal junction (TPJ) and Crus I of the cerebellum was revealed in the control group compared to the DCD group during the unpredictable timing condition. These findings suggest that the reported difficulties in timing in DCD may be due to under-activation in frontal, parietal and cerebellar areas.

\section{Summary}

While each of the six fMRI studies outlined report disparate findings in BOLD responses between children with and without DCD while they undertook motor tasks, there do appear to be some commonalities. Nevertheless, interpretation of these commonalities is problematic due to the difficulties of disentangling task-related activity from broader differences between groups, and 
because of the small sample sizes in each of the studies. In order to address these issues, Fuelscher and colleagues (2018) used a meta-analytic technique called activation likelihood estimation (ALE) to compare and summarise the overall activation patterns within each of these studies. This technique enables the authors to statistically establish which areas are reliably different between groups and across tasks. The authors found that the middle frontal gyrus, superior frontal gyrus, supramarginal gyrus, inferior parietal lobule, and parts of the cerebellum showed consistently lower activity in the DCD group when compared to controls. They also showed increased activity in thalamic areas in the DCD group. Aside from the cerebellum, none of the areas displaying reduced activity are directly involved in control of movement. They are, however, involved in higher functions related to planning and attention that do indirectly play a role in movement control. By combining the effects of multiple small studies to effectively boost the sample size to approximately 80 participants per group, this study provides the best evidence for the potential neural correlates of motor performance in DCD. However, while these findings are undoubtedly more robust than the findings of the individual studies included in the analysis, they should still be treated cautiously as they are likely to be affected by low power within the individual studies (Button et al., 2013; Nord et al., 2017) and publication bias (Jennings and Van Horn, 2012). Thus, rather than conclusive evidence for involvement of the aforementioned areas, this study should be treated as a robust starting point for future studies of neural correlates of motor performance in DCD.

All of the studies discussed used standardised tests to establish that their DCD groups have a degree of motor difficulty appropriate for the diagnosis. However, some found no differences between groups on their experimental tasks (Zwicker et al., 2010, 2011; Pangelinan, Hatfield and Clark, 2013). There are a number of explanations why no differences on these tasks were found, the most notable being: task difficulty, coping strategies, and individual differences in motor ability (Cantin, Ryan and Polatajko, 2014). While this presents some difficulty in interpreting the results of these studies, it does not entirely remove their value as evidence. Specifically, the inclusion of techniques to record neural activity allow for the identification of atypical patterns of processing 
during these tasks that may be associated with the features of DCD, even without behavioural differences (e.g. increased or decreased allocation of attentional resources). Nonetheless, until these studies have been replicated their findings should be interpreted cautiously.

It is important to note that, while the characteristic features of DCD are primarily motorbased, there have been a number of suggestions that problems either stem from, or are exacerbated by, cognitive difficulties. The next section examines the neuroscientific evidence available for these cognitive hypotheses.

\section{Cognitive hypotheses}

\section{Visual perception}

Based on evidence collected from behavioural tests, visual perception was an early factor thought to underlie the difficulties in DCD (Hulme, Smart and Moran, 1982; Lord and Hulme, 1987, 1988). Mon-Williams and colleagues (1996) addressed this hypothesis by examining the EEG activity elicited by the presentation of visual stimuli (also known as visually evoked potentials; VEPs). Although VEP amplitudes of early components for wider stimuli differed between DCD and control groups, the authors suggested that these differences were likely due to the presence of more noise in the recordings from the DCD group (due to inattention and movement). They concluded that lowlevel visual perception does not appear to play a role in the movement difficulties of DCD. These conclusions have been supported by other work investigating visual perception in DCD (MonWilliams, Pascal and Wann, 1994), and the ERP results have been replicated a number of times in subsequent EEG studies (e.g. Tsai et al., 2009). As noted by Mon-Williams and colleagues, however, these results only demonstrate that there are no deficits in low-level visual processing, and it may be that higher levels are affected. 


\section{Visuospatial Attention}

One suggestion for a higher level visual process that may be affected is visuospatial attention. That is, individuals with DCD display difficulties in visuospatial attention that may either cause or exacerbate the motor difficulties they experience (Wilson, Maruff and McKenzie, 1997; Wilson and McKenzie, 1998). Tsai and colleagues have conducted a number of EEG studies to explore the neural basis of these reported attention deficits in DCD (Tsai et al., 2009, 2010; Tsai, Wang and Tseng, 2012). All of these studies have examined ERPs associated with performance of a visuospatial cuing task. During this task a certain proportion of cues correctly indicate the position of a subsequent target (valid), a proportion incorrectly indicate the position of a subsequent target (invalid), and the rest have no cue. Valid cues reduce the time taken for participants to respond to the target, while there is no difference between target response times for invalid cue and no cue trials. In all of these studies the behavioural effects of cueing have been stable in both the TD and DCD groups, that is: the DCD group responded slower in all conditions and both groups benefited from the valid cues, but the benefit was smaller for the DCD group.

Tsai and colleagues have examined the mean amplitude and latency of ERP components across all of these studies, namely: N1, N2 and P3 following the target. N1 is an alternate name given to the previously described VEPs, and represents early visual processing. N2 and P3 components recorded from these areas are thought to represent higher visual attention/processing and response selection respectively. Across studies, the latency of the P3 component was longer in the invalid condition and the DCD group displayed smaller amplitude and later P3 components, regardless of condition. N2 latency was also longer in the DCD group for two of the studies (Tsai et al., 2009, 2010). After participating in a 10-week exercise programme (Tsai, Wang and Tseng, 2012), children with DCD demonstrated improvements in both motor performance and on the visuospatial attention task, there also appeared to be associated changes in P3 amplitude. However, analysis of post-intervention P3 amplitude revealed no differences between the intervention and two 
control groups. The authors did not report any further post-hoc tests, including comparisons between pre- and post- intervention P3 amplitude, making interpreting the effects of the training intervention on neural signatures of attention difficult.

This research group have also recently looked at oscillatory activity associated with attentional orienting (Wang et al., 2015). The gaze cueing task used in previous studies was repeated, and the behavioural results were largely the same (i.e., both groups benefited from valid cues but the control group benefited more). Theta-band (4 -7 Hz) EEG activity in frontal electrodes was analysed, given the evidence that activity in this band is associated with spatial orienting. The TD group showed increased power in the theta band for the cued conditions, regardless of cue, compared to the non-cued condition. The DCD group did not show any difference in theta band power for any of the three conditions, suggesting that the source of the theta-band activity produced during spatial orienting may be dysfunctional in DCD. The authors also reported negative correlations between theta power and reaction times; however, these were not corrected for multiple comparisons and should be interpreted cautiously.

\section{Executive function}

Recent evidence has suggested that individuals with DCD may have deficits in executive functioning, which are higher-order cognitive abilities used to regulate and control behaviour (Diamond, 2013; see Leonard and Hill, 2014, for a review). Several studies have used neuroimaging to investigate the neural underpinning of this potential executive dysfunction, focusing specifically on visuospatial working memory (VSWM) and inhibitory control.

Two studies have used EEG to assess VSWM in DCD. Both studies used an experimental paradigm where children were asked to compare the positions of stimuli within a grey box (Tsai et al., 2012; Wang et al., 2017). Stimuli were either presented together or after a delay. In the first study, Tsai and colleagues examined the amplitude and latency of the P3 and the positive slow wave (pSW; a component associated with information retrieval and response selection) components. The 
P3 amplitude in the response phase was smaller in the DCD group than in controls regardless of the condition, mirroring previously discussed results. Furthermore, the P3 amplitude was larger in the delay conditions than in the immediate condition for all groups, reflecting the reduced accuracy and longer response times after a delay. Finally, there was a significant interaction between group and condition for P3 amplitude, with the DCD group showing significantly smaller amplitudes in the delayed conditions than in the immediate condition, which related to less accurate and longer response times after a delay. The pSW amplitude was also significantly smaller in the DCD group, and in the immediate condition. The authors suggest that these results indicate that the DCD group allocated fewer neural resources during the stimulus evaluation and response selection phase, as indicated by the differences in P3 amplitude. In addition, the group differences in pSW illustrate a more general difficulty in the retrieval process phase for those with DCD.

In the second study, frontal theta band and parietal alpha band activity associated with VSWM was recorded in children with and without DCD (Wang et al., 2017). Frontal theta band activity showed a significant increase in the period directly after stimulus presentation in both conditions. While there were no group differences in the non-delayed condition, the DCD group displayed lower activity in the delayed condition, reduced alpha suppression in the posterior region immediately prior to recall, and reduced frontal theta activity for a brief period after recall. These findings provide some support for the previous study, with the DCD children showing atypical activity during the retrieval stage. They also provide some more insight into differences in VSWM processing in DCD, with diminished activity during the encoding and maintenance phases.

Two studies have also used fMRI to investigate inhibitory control in children with DCD (Querne et al., 2008; Thornton et al., 2018). Both studies used a Go-NoGo task to measure inhibition, in which participants must respond by pressing a button when they see one stimulus, and withhold this response when they see another stimulus. The number of errors differed between DCD and control children in both studies, although this was only the case for those with an additional diagnosis of attention deficit-hyperactivity disorder (ADHD) in the Thornton et al. study (co- 
occurring diagnoses were not controlled in the other study). Furthermore, Querne and colleagues reported significantly stronger connectivity between the inferior parietal cortex and both the middle frontal cortex and the anterior cingulate cortex in DCD compared to controls, which was bilateral but more pronounced in the left hemisphere, and decreased connectivity between the right striatum and parietal cortex in children with DCD. However, Thornton and colleagues reported no significant differences between DCD and control groups in BOLD activity during the task. From these studies, it therefore seems important to control for co-occurring diagnoses when investigating DCD.

\section{Internal modelling}

One of the main hypotheses grounded in models of motor control is that of the internal modelling deficit. This hypothesis suggests that the motor difficulties experienced by individuals with DCD are due to the inability of the motor system to generate accurate predictions about the consequences of planned actions, which affects its ability to update actions accordingly (Adams et al., 2014). Examining the internal model directly is difficult, but one of the accepted methods is through motor imagery, typically using a hand rotation task. This task asks participants to determine whether the hand presented on screen in a variety of rotations is a right or left hand.

Results have been mixed in relation to studies that have tried to investigate the neural underpinnings of this task. In terms of reaction times to respond to the stimulus, Lust and colleagues (2006) found no significant differences between children with and without DCD. Adult participants with probable DCD also demonstrated this pattern (Kashuk et al., 2017), while adults with a DCD diagnosis did perform worse than the control group overall (Hyde et al., 2018). These three studies used different techniques to assess neural activation during this task. Lust and colleagues reported no significant differences between groups in the amplitude or latency of the rotation-related negativity ERP component (observed in parietal electrodes 300-700 ms after stimulus onset), reflecting their behavioural results. Analysis of task-related fMRI activity by Kashuk and colleagues 
revealed that the control group showed significantly greater activity in the middle frontal gyrus bilaterally, the left superior parietal lobe, and lobule VI of the cerebellum as the task increased in difficulty, compared to the DCD group. Finally, Hyde and colleagues, using transcranial magnetic stimulation (TMS), revealed differences in the motor-cortical excitability of the two groups during the task. Specifically, the motor-cortical excitability significantly increased for the control group, but there was no change for the DCD group. This suggests that the primary motor cortex may not be engaged during motor imagery in the DCD group, providing a potential neural correlate for the internal modelling deficit hypothesis. The three studies taken together also highlight the importance of group choice (e.g., children or adults, those with a diagnosis or just screened for motor difficulties), and of the neuroimaging technique used to investigate the task at hand.

\section{Imitation and the mirror neuron system}

Related to the internal modelling deficit is the mirror neuron system (MNS), a network of areas in the brain thought to be used when an individual observes, executes, imagines, or imitates an action (Aziz-Zadeh et al., 2006; Iacoboni and Dapretto, 2006). As discussed by Iacoboni and Dapretto, the MNS network is thought to consist of the inferior frontal gyrus (IFG), ventral premotor cortex (vPM), inferior parietal lobule (IPL) and the superior temporal sulcus (STS). Alongside the behavioural evidence of motor imagery deficits in DCD, there is evidence that imitation is also impaired (Sinani, Sugden and Hill, 2011; Elbasan, Kayıhan and Duzgun, 2012; Reynolds, Kerrigan, et al., 2017).

Two studies have been conducted to test this hypothesis, both conducted by Reynolds and colleagues (Reynolds et al., 2015; Reynolds, Billington, et al., 2017) with children. The first study included three phases: action observation (viewing a finger sequencing task being executed), action execution (performing the task after viewing a still image of the first hand stimulus), and action imitation (performing the task while viewing it being executed). Comparison of whole brain activity between groups for each of the conditions revealed more activation in the IFG, bilateral pre-central 
gyri, and left middle temporal gyrus in the DCD group compared to controls during the observation condition. There were, however, no group differences in the imitation and execution conditions. Region of interest analyses focussed on those areas explicitly associated with the MNS revealed condition-specific differences in BOLD activity, and an interaction between condition and group at one site: the Pars Opercularis. This area is located in the IFG and has been shown to be active during action observation and imitation. The control group displayed greater activation than the DCD group in this area during the imitation condition, suggesting that it may not be properly recruited during action imitation in DCD. However, these differences were not statistically significant after correction for multiple comparisons, and it is difficult to interpret the results without the corresponding behavioural data. The authors also note that the overall lack of difference in activity between the imitation and execution conditions for the whole brain analysis precludes a clear interpretation. They speculate that this lack of difference may be due to learning effects from practice of the finger sequencing task and may have masked true between-group differences in activity.

Consequently, Reynolds and colleagues conducted a follow-up study that utilised a modified version of a finger tapping task that has been successfully used in previous research into the MNS (Reynolds, Billington, et al., 2017). This simply consisted of tapping the right index finger side-toside in time with a stimulus, allowing little room for learning effects. As with the previous study, the task was divided into observation, execution, and imitation conditions, but also added a motor imagery condition. As before, BOLD activity was analysed across the whole brain and in specific regions associated with the MNS, and only found weak evidence for differences between the groups in the different conditions. Taken together these studies seem to rule out deficits in the mirror neuron system as a potential explanation for the action imitation difficulties in DCD that have been reported. 


\section{Summary}

Investigation of the neural correlates of the cognitive hypotheses has provided evidence that rules out the visual perception and mirror neuron hypotheses. The remaining hypotheses have mixed evidence supporting them at best, with the majority requiring more exploration to resolve discrepancies between behavioural and neuroscientific findings. More recent research has therefore turned to considering structural and functional connectivity in DCD, which is outlined further below.

\section{Structural and functional connectivity in DCD}

\section{White matter structure}

Diffusion tensor imaging (DTI) uses the diffusion of water molecules in neural tissue to measure the integrity of axonal or white-matter tracts in the brain. A number of diffusion indexes can be measured using DTI, but the two most commonly reported are diffusivity and anisotropy (See Soares et al., 2013 for an in-depth description of DTI). Both of these measures quantify how freely the water molecules in the white-matter tracts can diffuse. Diffusivity provides a value for the magnitude of diffusion for each axis direction, although it is typically summarised by the mean diffusivity (MD): the mean magnitude of diffusivity across all three axes. Some studies also report axial (AD) and radial diffusivity (RD), which refer to the magnitude of diffusion along the tract and the magnitude of the diffusion in the two directions perpendicular to the tract, respectively. Anisotropy, or more specifically fractional anisotropy (FA), provides a normalised value for diffusivity across all three axis directions. Low values indicate free movement in all directions, while high values indicate that the molecules can only flow in one particular direction.

Three studies have explored the integrity of white-matter tracts in DCD using DTI. Zwicker and colleagues (2012) examined the major motor, sensory, and cerebellar white-matter pathways in 
16 children, 7 with DCD and 9 without. No significant differences in FA were reported between the groups for any of the tracts investigated. The DCD group did however show a lower MD than the control group in the corticospinal tract, seemingly driven by lower AD. Furthermore, participants' scores on a standardised motor assessment showed a positive correlation with AD in the motor and sensory tracts. Langevin and colleagues (2014) examined different white-matter tracts, focusing on the major inter-hemispheric and two intra-hemispheric pathways. Of the 84 children recruited for this study, only 9 had DCD without any co-occurring disorders. Unlike Zwicker and colleagues, the authors reported no between-group differences in MD, but did note lower FA in the DCD group in the posterior portion of the inter-hemispheric tract and the lateral portion of one of the intrahemispheric tracts. They also reported a significant correlation between scores on a standardised motor assessment and the FA of the posterior portion of the inter-hemispheric tract in the DCD group.

Finally, Debrabrant and colleagues (2016) examined 19 of the main white-matter tracts in children with and without DCD. Of these tracts only the retrolenticular limb of the internal capsule showed differences in the DCD group. Bilaterally, the retrolenticular limb showed greater RD in the DCD group, while the right retrolenticular limb also showed lower FA. Furthermore, in both groups, scores on a standardised test of visuomotor integration were positively correlated with FA in the left retrolenticular limb. Debrabrant and colleagues also constructed a structural connectivity network and examined differences in typical network metrics. The DCD group had lower mean clustering coefficients and global efficiency, suggesting that individuals with DCD have poorer specialisation of neural areas and weaker information transfer across the whole brain. Global efficiency was positively correlated with scores on the assessment of visual-motor skill, suggesting that the poorer information transfer across the brain may underlie the core features of DCD.

Together, these studies give some indication that differences in white matter integrity may relate to the core features of DCD, particularly given that all three demonstrate correlations between structural measures and behavioural outcomes. Nonetheless, the lack of consistency in the findings 
of the three studies, both in terms of areas and measures affected, mean that no clear link between DCD symptoms and specific white-matter deficits can be drawn.

\section{Grey matter structure}

Examining grey matter presents an alternative way to explore potential structural correlates of neurodevelopmental disorders such as DCD. This measure gives an indication of the degree of synaptic connectivity in an area; more grey matter suggests greater connectivity, and vice versa. The most common way of exploring this is to examine differences in the thickness of the grey matter in different regions of the brain.

Thus far, two studies have used this approach to explore differences in this between DCD and neurotypical individuals. Of the 28 cortical areas explored by Langevin and colleagues (2015), the group of children with DCD only showed thinning in the right temporal pole. The authors also reported several correlations between thickness of grey matter in particular regions and performance on behavioural tasks. These include correlations between the right caudal middle frontal cortex and an inhibition switching task, between the left precentral cortex and a response set task, and between the left entorhinal cortex and an auditory attention task. Strangely, they found no structuralbehavioural correlations for a task assessing motor performance, which would be expected for this group. Caeyenberghs and colleagues (2016) reported a difference between children with DCD and controls in the clustering coefficient in the lateral orbitofrontal cortex, indicating increased connectivity within that region. However, as the authors make clear, these results should be treated with caution as they were exploratory and not corrected for multiple comparisons. Finally, Reynolds, Licari and colleagues (2017) measured the volume of grey matter in a particular area, rather than just the thickness, thus taking into account differences in surface area and cortical folding. There were no significant differences in overall grey matter, white matter, or total intracranial volumes between groups of children with and without DCD. However, the authors did 
report that, compared to the control group, the DCD group had smaller relative grey matter volume in the right superior frontal and middle frontal gyri.

\section{Functional connectivity}

Alongside exploring the structural correlates of DCD, it is also possible to explore the functional connectivity by examining neural signals when the participant is not undertaking a specific task ('resting-state activity'). Resting-state activity is examined to see if fluctuations in the signal in different cortical areas correlate with one another, indicating the degree to which areas are functionally connected with each other.

Only two studies have looked at functional connectivity in children with DCD (McLeod et al., 2014, 2016). The first found that, compared to controls, the DCD group exhibited reduced functional connectivity between the primary motor cortex (M1) and a number of cortical areas. These included: the right frontal operculum cortex, right supramarginal gyrus, bilateral insular cortices, superior temporal gyri, bilateral caudate, right nucleus accumbens, pallidum, and putamen. The latter study examined which areas showed stronger functional connectivity in the right and left sensorimotor areas, respectively. The DCD group did not show the stronger functional connections between the right sensorimotor areas and thalamic and cerebellar areas seen in controls. Instead the opposite was revealed: stronger connections between the left sensorimotor areas and thalamic and cerebellar areas. The authors suggest that these results indicate a lack of hemispheric dominance in DCD, which may explain the bimanual coordination deficits seen in DCD.

Using TMS, He and colleagues (2018) assessed the intra-cortical inhibition and interhemispheric inhibition of M1 in young adults with and without DCD. This method provides a direct way of examining functional connectivity within and between the motor cortices. They found no difference in the intra-hemispheric measures. The DCD group did, however, have reduced interhemispheric inhibition compared to controls, and there was an association between interhemispheric inhibition and manual dexterity in a standardised motor battery. This suggests that the 
weaker inter-cortical inhibition observed may underlie bimanual coordination difficulties in DCD. However, this conclusion is tentative due to similar findings in ADHD (Richter et al., 2007) for which the authors did not screen despite it commonly co-occurring alongside DCD (Kaplan et al., 2006).

\section{Summary}

Overall the studies presented exploring structural and functional connectivity in DCD have provided some evidence for broader differences in the structure and connectivity of the brain in DCD. Nonetheless the support for any one specific cause is weak. Many of the studies presented are exploratory, yet they do not explicitly state if and how inflation of Type 1 errors from multiple comparisons was controlled. Furthermore, like much of the research outlined thus far, many of the studies presented have small sample sizes. The issue of small sample sizes is discussed in the following section alongside other limitations of the current research, potential solutions, and future directions.

\section{Current issues and future directions}

Within the literature outlined thus far there are a number of common issues that should be addressed in order to strengthen research into the neural underpinnings of DCD. This section will highlight two of the most pressing issues, briefly discuss their ramifications, and outline some suggestions for solutions.

\section{Small sample sizes}

A key issue in the broader field of cognitive neuroscience that has emerged over the last decade is that of the small sample sizes used within imaging studies. In the literature outlined in this chapter, 
the majority of studies do not exceed a cell size of 20 participants and none exceed 40 . This only gives these studies the ability to detect the largest of differences and also renders many of the brainbehaviour correlations difficult to interpret (Yarkoni, 2009).

In addition to the more general reasons for small sample sizes in cognitive neuroscience research (i.e. the time and resource cost of recruiting large numbers of participants), DCD-specific recruitment issues can also affect sample size. Despite the fact that the estimated prevalence of DCD in the general population makes it one of the more common developmental disorders (Bishop, 2010) the disorder itself is comparatively unknown amongst parents and teachers (Piek and Edwards, 1997). Consequently, the lack of familiarity with DCD in the public makes recruitment of large samples difficult. Additionally, subclinical symptoms of other disorders, such as increased tactile or auditory sensitivity, or inattention/hyperactivity, can affect the amount and quality of data that can be recorded through neuroimaging methods with individuals with DCD, meaning that final sample sizes can be reduced.

Ultimately, bringing DCD closer to the fore in public and professional consciousness is the solution to the problem of recruiting larger sample sizes. However, this will not happen overnight and so shorter-term solutions are needed. Establishing multi-lab studies is one such solution, reducing the need for a single laboratory to recruit many participants alone by spreading the burden across several different laboratories and ensuring that published research is better powered. Combining this approach with an OpenfMRI-like database would add further robustness to investigations into the neural correlates of DCD, allowing for replication of previous findings with larger sample sizes and exploration of hypotheses without needing additional data collection.

\section{Age range of samples}

Within the DCD literature discussed, the range of ages examined is relatively narrow (mainly 8-12 years old). This is not necessarily a problem on its own: ensuring that the age range of the sample collected for a particular study is constrained allows for more robust conclusions to be drawn about 
neural development within DCD at that time point, particularly with small sample sizes. However, it does limit the understanding of the broader trajectory of neuromotor development, the atypical trajectory of DCD, where these trajectories begin to diverge, and what happens to these trajectories in adulthood.

For adult samples, the main limiting factor is the lack of reliable tools for identifying DCD in adulthood. Specifically, none of the widely used motor ability assessments are standardised beyond 21 years (Hands, Licari and Piek, 2015), making fulfilment of criterion A difficult. For children under 5 years, there is variability in motor development, rate of acquisition of activities of daily living, motivation, and cooperation of children that makes giving a formal diagnosis unreliable in all but the most severe cases (Blank et al., 2012). This is compounded by the relative difficulty in collecting neural data from children below the age of 7 (Poldrack, Paré-Blagoev and Grant, 2002; Wilke et al., 2003; Yerys et al., 2009; Raschle et al., 2012).

The previous suggestions for increasing the sample sizes of DCD research would also allow for the broadening of age ranges included as part of that research. However, this would only extend the available age range to the limits of the available diagnostic tools (from 8-12 up to 7-17). Beyond this, either new diagnostic tools would have to be developed or existing ones would have to be validated with new age ranges and, even then, this does not account for the aforementioned variability in younger children.

Longitudinal approaches represent an alternative way of addressing this issue using the tools currently available. In the case of adults, it is possible to identify children and adolescents with DCD using the tools available and then follow them up when they enter adulthood. This would also allow exploration of neural features that may contribute to the persistence of DCD into adulthood. In the case of younger children, an “at-risk” longitudinal approach could be taken (e.g., identifying factors associated with an increased risk of developing the disorder). Two examples specific to DCD include: being related to someone who already has DCD, and premature birth (Martin, Piek and Hay, 2006; Edwards et al., 2011). Identifying and testing infants and toddlers who are at risk of 
DCD and following them up at an age when a diagnosis could be properly established would provide a window into the early neural motor development of DCD.

However, it should be noted that these approaches are likely to require considerable time and resources to achieve, especially as a large initial sample will need to be recruited in order to account for drop-outs and resolutions.

\section{Future directions}

The difficulty of collecting usable data from younger children with DCD also needs to be addressed, and to accomplish this we have to turn to newer neuroscientific techniques. Functional near-infrared spectroscopy (fNIRS) presents an alternative to EEG and fMRI. This technique is able to record BOLD activity from shallow cortical areas without the need for an MRI scanner. In addition, because it consists of head mounted sensors (much like EEG), it is much less affected by head movements than either fMRI or EEG. Indeed, it has been successfully used in studies that look at the BOLD responses in infants (Nishiyori, 2016). fNIRS is not without its limitations, however, the most notable of which is the poorer spatial resolution compared to fMRI: it is unable to provide the direct link between activity and structure available from fMRI. Despite this, combining a technique such as fNIRS with an at-risk recruitment approach provides an excellent way of examining the neural correlates of DCD in younger children.

Other newer neuroscientific techniques present novel opportunities to deepen our understanding of the neural basis of DCD. The two most notable of these are: Non-invasive brain stimulation and magnetic resonance spectroscopic imaging (MRSI)

Brain stimulation techniques are already being used to explore the potential involvement of the motor cortex in DCD (e.g. He et al., 2018; Hyde et al., 2018). There is the scope, however, to expand into using TMS to examine the involvement of other cortical areas, especially as TMS can be used to establish a causal relationship between a specific behaviour and an area of the cortex (Walsh and Cowey, 2000). Furthermore, forms of transcranial electric stimulation have been used to 
alter the excitability of areas of the cortex to modulate behavioural performance in a range of experimental paradigms and with a variety of participants (Kuo and Nitsche, 2012).

In contrast, MRSI has not yet been employed with DCD samples. MRSI extends the principles of MRI to detect signals from a number of other molecules, including neurotransmitters. This potentially allows for a more specific understanding of the neural underpinnings of DCD; rather than simply concluding that a specific brain area is involved, it may be possible to implicate a specific type of neuron based on differences in neurotransmitter concentrations. An excellent example of this is work conducted by Stagg and colleagues looking at the role of GABA in M1 and motor learning (Stagg, Bachtiar and Johansen-Berg, 2011; Stagg, 2014). However, given that this technique can detect concentrations of multiple molecules in a single scan, it adds another dimension to already complex MRI data sets. Without strong, theory-driven hypotheses for the involvement of a particular neurotransmitter, this increases the risk of false positives. Based on the findings outlined here, this theoretical basis does not yet exist for DCD.

\section{Conclusions}

While some of the studies presented in this chapter have provided evidence to rule out certain hypotheses, overall there is no clear consensus about the neural underpinnings of DCD from the current literature. There is, however, some evidence for the involvement of a number of areas, including: the cerebellum, the basal ganglia, the parietal lobe, and parts of the frontal lobe. In addition, there is speculative evidence for the involvement of white matter pathways in the disorder. A clearer picture of the developmental cognitive neuroscience of DCD is unlikely to emerge unless some of the major issues in the field are addressed by future research. Sharing data to increase the size of datasets, taking a developmental perspective, and making use of a range of new methods as part of a theory-led approach will be key to developing our understanding of the underlying mechanisms involved in DCD. Given the prevalence of the disorder and its impact on both motor 
and non-motor domains, this could have a significant impact on the lives of those with DCD, as well as on the knowledge of researchers and practitioners. 


\section{References}

Adams, I. L. J. et al. (2014) 'Compromised motor control in children with DCD: A deficit in the internal model?-A systematic review’, Neuroscience \& Biobehavioral Reviews, 47, pp. 225-244. doi: 10.1016/j.neubiorev.2014.08.011.

American Psychiatric Association (2013) Diagnostic and Statistical Manual of Mental Disorders, Fifth Edition. 5th edition edition. American Psychiatric Publishing.

Aziz-Zadeh, L. et al. (2006) 'Lateralization of the Human Mirror Neuron System', Journal of Neuroscience, 26(11), pp. 2964-2970. doi: 10.1523/JNEUROSCI.2921-05.2006.

Biotteau, M. et al. (2017) 'Neural changes associated to procedural learning and automatization process in Developmental Coordination Disorder and/or Developmental Dyslexia', European Journal of Paediatric Neurology, 21(2), pp. 286-299. doi: 10.1016/j.ejpn.2016.07.025.

Bishop, D. V. M. (2010) 'Which Neurodevelopmental Disorders Get Researched and Why?', PLoS ONE, 5(11), p. e15112. doi: 10.1371/journal.pone.0015112.

Blank, R. et al. (2012) 'European Academy for Childhood Disability (EACD): Recommendations on the definition, diagnosis and intervention of developmental coordination disorder (long version)', Developmental Medicine and Child Neurology, 54(1), pp. 54-93. doi: 10.1111/j.14698749.2011.04171.x.

Button, K. S. et al. (2013) 'Power failure: why small sample size undermines the reliability of neuroscience’, Nature Reviews Neuroscience, 14(5), pp. 365-376. doi: 10.1038/nrn3475.

Caeyenberghs, K. et al. (2016) 'Neural signature of developmental coordination disorder in the structural connectome independent of comorbid autism', Developmental Science, 19(4), pp. 599612. doi: 10.1111/desc.12424.

Cairney, J. et al. (2005) 'Developmental coordination disorder, generalized self-efficacy toward physical activity, and participation in organized and free play activities.', The Journal of pediatrics, 147(4), pp. 515-20. doi: 10.1016/j.jpeds.2005.05.013.

Cantin, N., Ryan, J. and Polatajko, H. (2014) 'Impact of task difficulty and motor ability on visualmotor task performance of children with and without developmental coordination disorder.', Human movement science, 34, pp. 217-32. doi: 10.1016/j.humov.2014.02.006.

de Castelnau, P. et al. (2008) 'A study of EEG coherence in DCD children during motor synchronization task', Human Movement Science, 27(2), pp. 230-241. doi: 10.1016/j.humov.2008.02.006.

Cousins, M. and Smyth, M. M. (2003) 'Developmental coordination impairments in adulthood', Human Movement Science, 22(4-5), pp. 433-459.

Debrabant, J. et al. (2013) 'Neural underpinnings of impaired predictive motor timing in children with Developmental Coordination Disorder’, Research in Developmental Disabilities, 34(5), pp. 1478-1487. doi: 10.1016/j.ridd.2013.02.008. 
Debrabant, J. et al. (2016) 'Brain Connectomics of Visual-Motor Deficits in Children with Developmental Coordination Disorder', The Journal of Pediatrics, 169, pp. 21-27.e2. doi: 10.1016/j.jpeds.2015.09.069.

Diamond, A. (2013) 'Executive functions', Annual Review of Psychology, 64, pp. 135-168. doi: 10.1146/annurev-psych-113011-143750.

Edwards, J. et al. (2011) 'Developmental Coordination Disorder in School-Aged Children Born Very Preterm and/or at Very Low Birth Weight: A Systematic Review', Journal of Developmental \& Behavioral Pediatrics, 32(9), pp. 678-687. doi: 10.1097/DBP.0b013e31822a396a.

Elbasan, B., Kayıhan, H. and Duzgun, I. (2012) 'Sensory integration and activities of daily living in children with developmental coordination disorder', Italian Journal of Pediatrics, 38, p. 14. doi: 10.1186/1824-7288-38-14.

Faught, B. E. et al. (2005) 'Increased risk for coronary vascular disease in children with developmental coordination disorder', Journal of Adolescent Health, 37(5), pp. 376-380. doi: 10.1016/j.jadohealth.2004.09.021.

Fuelscher, I. et al. (2018) 'Differential activation of brain areas in children with developmental coordination disorder during tasks of manual dexterity: An ALE meta-analysis', Neuroscience \& Biobehavioral Reviews, 86, pp. 77-84. doi: 10.1016/j.neubiorev.2018.01.002.

Geuze, R. H. (2003) 'Static balance and developmental coordination disorder', Human Movement Science, 22(4-5), pp. 527-548. doi: 10.1016/j.humov.2003.09.008.

Gheysen, F., Van Waelvelde, H. and Fias, W. (2011) 'Impaired visuo-motor sequence learning in Developmental Coordination Disorder', Research in Developmental Disabilities, 32(2), pp. 749756. doi: 10.1016/j.ridd.2010.11.005.

Gubbay, S. S. et al. (1965) 'Clumsy children: A study of apraxia and agnosic deficits in 21 children’, Brain, 88, pp. 295-312.

Hands, B., Licari, M. and Piek, J. (2015) 'A review of five tests to identify motor coordination difficulties in young adults', Research in Developmental Disabilities, 41-42, pp. 40-51. doi: 10.1016/j.ridd.2015.05.009.

He, J. L. et al. (2018) 'Interhemispheric Cortical Inhibition Is Reduced in Young Adults With Developmental Coordination Disorder’, Frontiers in Neurology, 9. doi: 10.3389/fneur.2018.00179.

Hulme, C., Smart, A. and Moran, G. (1982) 'Visual perceptual deficits in clumsy children', Neuropsychologia, 20(4), pp. 475-481. doi: 10.1016/0028-3932(82)90046-X.

Hyde, C. et al. (2018) 'Corticospinal excitability during motor imagery is reduced in young adults with developmental coordination disorder', Research in Developmental Disabilities, 72, pp. 214224. doi: 10.1016/j.ridd.2017.11.009.

Hyde, C. and Wilson, P. (2013) 'Impaired Online Control in Children With Developmental Coordination Disorder Reflects Developmental Immaturity', Developmental Neuropsychology, 38(2), pp. 81-97. doi: 10.1080/87565641.2012.718820.

Iacoboni, M. and Dapretto, M. (2006) 'The mirror neuron system and the consequences of its dysfunction’, Nature Reviews Neuroscience, 7(12), pp. 942-951. doi: 10.1038/nrn2024. 
Jennings, R. G. and Van Horn, J. D. (2012) 'Publication bias in neuroimaging research: Implications for meta-analyses’, Neuroinformatics, 10(1), pp. 67-80. doi: 10.1007/s12021-011-9125-y.

Kagerer, F. A. et al. (2004) 'Visuomotor adaptation in children with developmental coordination disorder’, Motor Control, 8(4), pp. 450-460.

Kagerer, F. A. et al. (2006) 'Abrupt, but not gradual visuomotor distortion facilitates adaptation in children with developmental coordination disorder’, Human Movement Science, 25(4-5), pp. 622633. doi: 10.1016/j.humov.2006.06.003.

Kaplan, B. et al. (2006) 'Comorbidity, co-occurrence, continuum: What's in a name?', Child: Care, Health and Development, 32(6), pp. 723-731. doi: 10.1111/j.1365-2214.2006.00689.x.

Kashiwagi, M. et al. (2009) 'Parietal dysfunction in developmental coordination disorder: a functional MRI study':, NeuroReport, 20(15), pp. 1319-1324. doi: 10.1097/WNR.0b013e32832f4d87.

Kashuk, S. R. et al. (2017) 'Diminished motor imagery capability in adults with motor impairment: An fMRI mental rotation study', Behavioural Brain Research, 334, pp. 86-96. doi: 10.1016/j.bbr.2017.06.042.

Kirby, A., Sugden, D. and Purcell, C. (2014) 'Diagnosing developmental coordination disorders.', Archives of disease in childhood, 99(3), pp. 292-6. doi: 10.1136/archdischild-2012-303569.

Kuo, M.-F. and Nitsche, M. A. (2012) 'Effects of Transcranial Electrical Stimulation on Cognition', Clinical EEG and Neuroscience, 43(3), pp. 192-199. doi: 10.1177/1550059412444975.

Langevin, L. M. et al. (2014) 'Common White Matter Microstructure Alterations in Pediatric Motor and Attention Disorders', The Journal of Pediatrics, 164(5), pp. 1157-1164.e1. doi: 10.1016/j.jpeds.2014.01.018.

Langevin, L. M., Macmaster, F. P. and Dewey, D. (2015) 'Distinct patterns of cortical thinning in concurrent motor and attention disorders', Developmental Medicine and Child Neurology, 57(3), pp. 257-264. doi: 10.1111/dmcn.12561.

Lejeune, C. et al. (2013) 'Intact procedural motor sequence learning in developmental coordination disorder', Research in Developmental Disabilities, 34(6), pp. 1974-1981. doi: 10.1016/j.ridd.2013.03.017.

Leonard, H. C. et al. (2015) 'Executive Functioning, Motor Difficulties, and Developmental Coordination Disorder', Developmental Neuropsychology, 40(4), pp. 201-215. doi: 10.1080/87565641.2014.997933.

Leonard, H. C. and Hill, E. L. (2014) 'Review: The impact of motor development on typical and atypical social cognition and language: A systematic review', Child and Adolescent Mental Health, 19(3), pp. 163-170. doi: 10.1111/camh.12055.

Leonard, H. C. and Hill, E. L. (2015) 'Executive Difficulties in Developmental Coordination Disorder: Methodological Issues and Future Directions', Current Developmental Disorders Reports, 2(2), pp. 141-149. doi: 10.1007/s40474-015-0044-8. 
Licari, M. K. et al. (2015) 'Cortical functioning in children with developmental coordination disorder: a motor overflow study.', Experimental brain research, 233(6), pp. 1703-10. doi: 10.1007/s00221-015-4243-7.

Licari, M. and Larkin, D. (2008) 'Increased associated movements: Influence of attention deficits and movement difficulties', Human Movement Science. (Developmental Coordination Disorder), 27(2), pp. 310-324. doi: 10.1016/j.humov.2008.02.013.

Licari, M., Larkin, D. and Miyahara, M. (2006) 'The influence of developmental coordination disorder and attention deficits on associated movements in children', Human Movement Science. (Approaches to Sensory-Motor Development in Infants and Children), 25(1), pp. 90-99. doi: 10.1016/j.humov.2005.10.012.

Lingam, R. et al. (2009) 'Prevalence of Developmental Coordination Disorder Using the DSM-IV at 7 Years of Age: A UK Population-Based Study’, Pediatrics, 123(4), pp. e693-e700. doi: 10.1542/ peds.2008-1770.

Lord, R. and Hulme, C. (1987) 'Perceptual judgements of normal and clumsy children', Developmental medicine and child neurology Child Neurology, 29(2), pp. 250-257. doi: 10.1111/j.1469-8749.1987.tb02143.x.

Lord, R. and Hulme, C. (1988) 'Visual perception and drawing ability in clumsy and normal children.', British Journal of Developmental Psychology, 6(1), pp. 1-9. doi: 10.1111/j.2044835X.1988.tb01075.x.

Lust, J. M. et al. (2006) 'An EEG study of mental rotation-related negativity in children with Developmental Coordination Disorder', Child: Care, Health and Development, 32(6), pp. 649-663. doi: 10.1111/j.1365-2214.2006.00683.x.

Martin, N. C., Piek, J. P. and Hay, D. (2006) 'DCD and ADHD: A genetic study of their shared aetiology', Human Movement Science. (Approaches to Sensory-Motor Development in Infants and Children), 25(1), pp. 110-124. doi: 10.1016/j.humov.2005.10.006.

McLeod, K. R. et al. (2014) 'Functional connectivity of neural motor networks is disrupted in children with developmental coordination disorder and attention-deficit/hyperactivity disorder', NeuroImage: Clinical, 4, pp. 566-575. doi: 10.1016/j.nicl.2014.03.010.

McLeod, K. R. et al. (2016) 'Atypical within- and between-hemisphere motor network functional connections in children with developmental coordination disorder and attention-deficit/hyperactivity disorder', NeuroImage: Clinical, 12, pp. 157-164. doi: 10.1016/j.nicl.2016.06.019.

Miller, L. T. et al. (2001) 'Clinical Description of Children with Developmental Coordination Disorder', Canadian Journal of Occupational Therapy, 68(1), pp. 5-15. doi: 10.1177/000841740106800101.

Miyahara, M. and Piek, J. (2006) 'Self-Esteem of Children and Adolescents with Physical Disabilities: Quantitative Evidence from Meta-Analysis’, Journal of Developmental and Physical Disabilities, 18(3), pp. 219-234. doi: 10.1007/s10882-006-9014-8. 
Mon Williams, M. A. et al. (1996) 'Visual evoked potentials in children with developmental coordination disorder', Ophthalmic and Physiological Optics, 16(2), pp. 178-183. doi: 10.1046/j.1475-1313.1996.95000364.x.

Mon-Williams, M., Pascal, E. and Wann, J. P. (1994) 'Ophthalmic factors in developmental coordination disorder’, Adapted Physical Activity Quarterly, 11(2), pp. 170-178.

Nishiyori, R. (2016) 'fNIRS: An Emergent Method to Document Functional Cortical Activity during Infant Movements’, Frontiers in Psychology, 7. doi: 10.3389/fpsyg.2016.00533.

Nord, C. L. et al. (2017) 'Power-up: A Reanalysis of "Power Failure" in Neuroscience Using Mixture Modeling', Journal of Neuroscience, 37(34), pp. 8051-8061. doi: 10.1523/JNEUROSCI.3592-16.2017.

Pangelinan, M. M., Hatfield, B. D. and Clark, J. E. (2013) 'Differences in movement-related cortical activation patterns underlying motor performance in children with and without developmental coordination disorder', Journal of Neurophysiology, 109(12), pp. 3041-3050. doi: 10.1152/jn.00532.2012.

Piek, J. et al. (2007) 'Depressive symptomatology in child and adolescent twins with attentiondeficit hyperactivity disorder and/or developmental coordination disorder.', Twin research and human genetics, 10(4), pp. 587-596. doi: 10.1375/twin.10.4.587.

Piek, J. P. and Edwards, K. (1997) 'The identification of children with developmental coordination disorder by class and physical education teachers', British Journal of Educational Psychology, 67(1), pp. 55-67. doi: 10.1111/j.2044-8279.1997.tb01227.x.

Poldrack, R. A., Paré-Blagoev, E. J. and Grant, P. E. (2002) 'Pediatric Functional Magnetic Resonance Imaging: Progress and Challenges’, Topics in Magnetic Resonance Imaging, 13(1), p. 61.

Pratt, M. L. and Hill, E. L. (2011) 'Anxiety profiles in children with and without developmental coordination disorder', Research in Developmental Disabilities, 32(4), pp. 1253-1259. doi: 10.1016/j.ridd.2011.02.006.

Querne, L. et al. (2008) 'Dysfunction of the attentional brain network in children with Developmental Coordination Disorder: A fMRI study', Brain Research, 1244, pp. 89-102. doi: 10.1016/j.brainres.2008.07.066.

Raschle, N. et al. (2012) 'Pediatric neuroimaging in early childhood and infancy: challenges and practical guidelines', Annals of the New York Academy of Sciences, 1252, pp. 43-50. doi: 10.1111/j.1749-6632.2012.06457.x.

Reynolds, J. E. et al. (2015) 'Mirror neuron activation in children with developmental coordination disorder: A functional MRI study', International Journal of Developmental Neuroscience, 47, pp. 309-319. doi: 10.1016/j.ijdevneu.2015.10.003.

Reynolds, J. E., Billington, J., et al. (2017) 'Mirror neuron system activation in children with developmental coordination disorder: A replication functional MRI study', Research in Developmental Disabilities. doi: 10.1016/j.ridd.2017.11.012. 
Reynolds, J. E., Kerrigan, S., et al. (2017) 'Poor Imitative Performance of Unlearned Gestures in Children with Probable Developmental Coordination Disorder’, Journal of Motor Behavior, 49(4), pp. 378-387. doi: 10.1080/00222895.2016.1219305.

Reynolds, J. E., Licari, M. K., et al. (2017) 'Reduced relative volume in motor and attention regions in developmental coordination disorder: A voxel-based morphometry study', International Journal of Developmental Neuroscience, 58, pp. 59-64. doi: 10.1016/j.ijdevneu.2017.01.008.

Richter, M. M. et al. (2007) 'Cortical excitability in adult patients with attention-deficit/hyperactivity disorder (ADHD)', Neuroscience Letters, 419(2), pp. 137-141. doi: 10.1016/j.neulet.2007.04.024.

Rosenblum, S. and Regev, N. (2013) 'Timing abilities among children with developmental coordination disorders (DCD) in comparison to children with typical development', Research in Developmental Disabilities, 34(1), pp. 218-227. doi: 10.1016/j.ridd.2012.07.011.

Sinani, C., Sugden, D. and Hill, E. L. (2011) 'Gesture production in school vs. clinical samples of children with Developmental Coordination Disorder (DCD) and typically developing children.', Research in developmental disabilities, 32(4), pp. 1270-82. doi: 10.1016/j.ridd.2011.01.030.

Skinner, R. A. and Piek, J. (2001) 'Psychosocial implications of poor motor coordination in children and adolescents', Human Movement Science, 20(1-2), pp. 73-94. doi: 10.1016/S01679457(01)00029-X.

Smits-Engelsman, B., Niemeijer, A. S. and Van Galen, G. P. (2001) 'Fine motor deficiencies in children diagnosed as DCD based on poor grapho-motor ability', Human Movement Science, 20(12), pp. 161-182. doi: 10.1016/S0167-9457(01)00033-1.

Soares, J. M. et al. (2013) 'A hitchhiker's guide to diffusion tensor imaging', Frontiers in Neuroscience, 7. doi: 10.3389/fnins.2013.00031.

Soares, J. M. et al. (2016) 'A Hitchhiker's Guide to Functional Magnetic Resonance Imaging', Frontiers in Neuroscience, 10. doi: 10.3389/fnins.2016.00515.

Stagg, C. J. (2014) 'Magnetic Resonance Spectroscopy as a tool to study the role of GABA in motor-cortical plasticity’, NeuroImage, 86, pp. 19-27. doi: 10.1016/j.neuroimage.2013.01.009.

Stagg, C. J., Bachtiar, V. and Johansen-Berg, H. (2011) 'The role of GABA in human motor learning’, Current Biology, 21(6), pp. 480-484. doi: 10.1016/j.cub.2011.01.069.

Summers, J., Larkin, D. and Dewey, D. (2008) 'Activities of daily living in children with developmental coordination disorder: Dressing, personal hygiene, and eating skills', Human Movement Science, 27(2), pp. 215-229. doi: 10.1016/j.humov.2008.02.002.

Thornton, S. et al. (2018) 'Functional brain correlates of motor response inhibition in children with developmental coordination disorder and attention deficit/hyperactivity disorder', Human Movement Science, 59, pp. 134-142. doi: 10.1016/j.humov.2018.03.018.

Tsai, C.-L. et al. (2009) 'Mechanisms of deficit of visuospatial attention shift in children with developmental coordination disorder: A neurophysiological measure of the endogenous Posner paradigm', Brain and Cognition, 71(3), pp. 246-258. doi: 10.1016/j.bandc.2009.08.006. 
Tsai, C.-L. et al. (2010) 'Deficits of visuospatial attention with reflexive orienting induced by eyegazed cues in children with developmental coordination disorder in the lower extremities: An eventrelated potential study', Research in Developmental Disabilities, 31(3), pp. 642-655. doi: 10.1016/j.ridd.2010.01.003.

Tsai, C.-L. et al. (2012) 'The neurophysiological performance of visuospatial working memory in children with developmental coordination disorder: Visuospatial Working Memory in Children with DCD', Developmental Medicine \& Child Neurology, 54(12), pp. 1114-1120. doi: 10.1111/j.14698749.2012.04408.x.

Tsai, C.-L., Wang, C.-H. and Tseng, Y.-T. (2012) 'Effects of exercise intervention on event-related potential and task performance indices of attention networks in children with developmental coordination disorder', Brain and Cognition, 79(1), pp. 12-22. doi: 10.1016/j.bandc.2012.02.004.

Wang, C.-H. et al. (2015) 'Frontal midline theta as a neurophysiological correlate for deficits of attentional orienting in children with developmental coordination disorder: Theta oscillation, attentional orienting, and DCD', Psychophysiology, 52(6), pp. 801-812. doi: 10.1111/psyp.12402.

Wang, C.-H. et al. (2017) 'Neural Oscillation Reveals Deficits in Visuospatial Working Memory in Children With Developmental Coordination Disorder', Child Development, 88(5), pp. 1716-1726. doi: $10.1111 /$ cdev.12708.

Watson, L. and Knott, F. (2006) 'Self-Esteem and Coping in Children with Developmental Coordination Disorder', The British Journal of Occupational Therapy, 69(10), pp. 450-456. doi: 10.1177/030802260606901003.

Wilke, M. et al. (2003) 'Functional magnetic resonance imaging in pediatrics', Neuropediatrics, 34(5), pp. 225-233. doi: 10.1055/s-2003-43260.

Wilson, P. et al. (2012) 'Understanding performance deficits in developmental coordination disorder: A meta-analysis of recent research', Developmental Medicine and Child Neurology, pp. 20-23. doi: 10.1111/j.1469-8749.2012.04436.x.

Wilson, P. H. et al. (2017) 'Cognitive and neuroimaging findings in developmental coordination disorder: new insights from a systematic review of recent research', Developmental Medicine and Child Neurology, 59(11), pp. 1117-1129. doi: 10.1111/dmcn.13530.

Wilson, P. H. and McKenzie, B. E. (1998) 'Information processing deficits associated with developmental coordination disorder: a meta-analysis of research findings', Journal of Child Psychology and Psychiatry, and Allied Disciplines, 39(6), pp. 829-840.

Wilson, P., Maruff, P. and McKenzie, B. E. (1997) 'Covert orienting of visuospatial attention in children with developmental coordination disorder', Developmental medicine and child neurology Child Neurology, 39(11), pp. 736-745. doi: 10.1111/j.1469-8749.1997.tb07375.x.

Wocadlo, C. and Rieger, I. (2008) 'Motor impairment and low achievement in very preterm children at eight years of age', Early Human Development, 84(11), pp. 769-776. doi: 10.1016/j.earlhumdev.2008.06.001.

Yarkoni, T. (2009) 'Big Correlations in Little Studies: Inflated fMRI Correlations Reflect Low Statistical Power-Commentary on Vul et al. (2009)

Big Correlations in Little Studies: Inflated fMRI Correlations Reflect Low Statistical Power- 
Commentary on Vul et al. (2009)', Perspectives on Psychological Science, 4(3), pp. 294-298. doi: 10.1111/j.1745-6924.2009.01127.x.

Yerys, B. E. et al. (2009) 'The fMRI success rate of children and adolescents: typical development, epilepsy, attention deficit/hyperactivity disorder, and autism spectrum disorders', Human brain mapping, 30(10), pp. 3426-3435. doi: 10.1002/hbm.20767.

Zwicker, J. G. et al. (2010) 'Brain Activation of Children With Developmental Coordination Disorder Is Different Than Peers’, Pediatrics, 126(3), pp. e678-e686. doi: 10.1542/peds.2010-0059.

Zwicker, J. G. et al. (2011) 'Brain activation associated with motor skill practice in children with developmental coordination disorder: an fMRI study’, International Journal of Developmental Neuroscience, 29(2), pp. 145-152. doi: 10.1016/j.ijdevneu.2010.12.002.

Zwicker, J. G. et al. (2012) 'Developmental Coordination Disorder: A Pilot Diffusion Tensor Imaging Study', Pediatric Neurology, 46(3), pp. 162-167. doi: 10.1016/j.pediatrneurol.2011.12.007. 